\title{
Hongos solubilizadores de fosfato en suelo de páramo cultivado con papa (Solanum tuberosum)
}

\author{
Phosphate Solubilizing Fungi in Paramo Soil Cultivated with Potato (Solanum \\ tuberosum)
}

M. E. Beltrán Pineda ${ }^{\mathrm{a}, *}$

Recepción: 14-ene-14

Aceptación: 16-oct-14

\begin{abstract}
Resumen
Los hongos solubilizadores de fosfato (HSF) son un grupo funcional de microorganismos que juegan un rol fundamental en el ciclaje de fósforo en los suelos; gracias a su actividad, las plantas pueden aprovechar las grandes reservas de fósforo insoluble que se encuentra fijado a los minerales del suelo, contribuyendo a que disminuya la aplicación de fertilizantes fosforados a los suelos. En este estudio se lograron aislar 43 cepas de hongos con capacidad solubilizadora de fosfato a partir de la rizósfera de cultivos de papa (Solanum tuberosum) establecidos en el páramo de Rabanal. Tras el proceso de evaluación cualitativa y cuantitativa de la capacidad solubilizadora de fosfato de los aislamientos, se obtuvieron dos cepas fúngicas con potencial biofertilizante, pertenecientes a los géneros Scopuraliopsis sp. y Penicillum sp., que podrían ser la base para la formulación de biofertilizantes. La aplicación de dichos insumos en los suelos constituye una estrategia de fertilización sostenible, que contribuye a la mitigación de la contaminación de zonas estratégicas como los páramos.
\end{abstract}

Palabras clave: Biofertilización, Hongos solubilizadores de fosfato, Suelo de páramo.

\begin{abstract}
The Phosphate Solubilizing Fungi (PSF) is a functional group of microorganisms that play a fundamental role in the cycling of phosphorus in soils; due to its activity, plants can take advantage of the large reserves of insoluble phosphorus that is attached to mineral soil, contributing to the decrease of the application of phosphorus fertilizers. In this study we were able to isolate 43 fungal strains with phosphate solubilizing capacity from the rhizosphere of potato crops (Solanum tuberosum) set in the paramo of Rabanal. After the process of qualitative and quantitative assessment of the phosphate solubilizing capacity of two fungal isolated potential biofertilizer strains, belonging to the genera Scopuraliopsis sp. and Penicillium sp. were obtained, that could be the basis for the formulation of biofertilizers. The application of these inputs in soils, constitutes a strategy for sustainable fertilization, contributing to the alleviation of contamination in strategic areas as the paramo.
\end{abstract}

Key words: Biofertilization, Phosphate Solubilizing Fungi, Paramo Soil.

${ }^{a}$ Departamento de Biología y Microbiología. Grupo de investigación Gestión ambiental. Facultad de Ciencias e Ingeniería. Universidad de Boyacá.

*Autor de correspondencia: mebeltranp@uniboyaca.edu.co 


\section{Introducción}

Boyacá, departamento cuya actividad económica predominante es la agricultura, es el segundo productor de papa (Solanum tuberosum) a nivel nacional, después de Cundinamarca, con una participación, en promedio, del $25.8 \%$ [1]; pero la agricultura intensiva en el departamento ha propiciado que grandes áreas estratégicas se vean degradadas por el deterioro de sus suelos, debido a la acumulación de insumos químicos [2]. Dentro de dichas áreas se encuentra el páramo de Rabanal, que a pesar de tener suelos que se caracterizan por su fuerte acidez y bajos niveles de fertilidad [3], condiciones que los hacen poco recomendables para actividades agrícolas intensivas, presenta grandes áreas cultivadas con papa, las cuales son fertilizadas con químicos por los agricultores con el objetivo de minimizar los bajos niveles de fertilidad inherentes e incrementar la productividad.

Dentro de los fertilizantes químicos aplicados en estos agroecosistemas se destacan los fosforados, que una vez aplicados a los suelos son inmovilizados y fijados, en gran parte, por óxidos libres o hidróxidos de aluminio, hierro o calcio, haciéndolos insolubles y no disponibles para las plantas [4]. Esta situación hace que sea necesaria la creación de variedades vegetales altamente eficientes en su adquisición y uso del nutriente [5] o la implementación de estrategias más sostenibles de fertilización.

Como se conoce, las interacciones microbianas con las raíces tienen un efecto profundo en el estatus nutricional de las plantas [6]; basados en ese principio se han desarrollado insumos biológicos, denominados biofertilizantes, que son reconocidos como enmiendas microbianas producidas a partir de microorganismos que viven normalmente en el suelo y que gracias a su actividad biológica pueden poner a disposición de las plantas una parte importante de sustancias nutritivas necesarias para su desarrollo. Se ha determinado que la aplicación de biofertilizantes no perjudica la calidad de los suelos, y contribuye a prácticas de fertilización más rentables y seguras para el medio ambiente [7].

Dentro de los microorganismos que pueden servir como base para la formulación de dichos insumos se incluyen los solubilizadores de fosfato (MSF); este grupo funcional de microorganismos está involucrado en un amplio rango de procesos que afectan la transformación del fósforo, siendo un componente integral del ciclo edáfico de este nutriente [8]. Los MSF incluyen cepas bacterianas y fúngicas. Los hongos, por constituir la mayor parte de la biomasa del suelo y ser abundantes en suelos ácidos, como los del páramo de Rabanal, fueron el objeto de estudio en esta investigación.

Debido a que los estudios microbiológicos en este tipo de ecosistemas son escasos y a que es urgente formular estrategias que minimicen la degradación del páramo, el objetivo de esta investigación fue aislar y caracterizar hongos solubilizadores de fosfato de la rizósfera de cultivos de papa (Solanum tuberosum) en suelos de páramo, con el fin de encontrar las cepas fúngicas más promisorias con potencial biofertilizante que podrán ser la base para la futura formulación de estos insumos, cuya aplicación podría mitigar en gran medida los niveles de degradación de suelos en estas áreas protegidas.

\section{Metodología}

\subsection{Obtención de las muestras de suelo}

El muestreo de suelos se realizó en la vereda San José del Gacal, del municipio de Ventaquemada- Boyacá, que está localizada en inmediaciones del páramo del Rabanal, a una altura de 3200 msnm. Estos suelos se caracterizan por presentar fuerte acidez y bajos niveles de fertilidad [3]. Se tomaron muestras de cuatro fincas cultivadas con papa (Solanum tuberosum), las cuales se recolectaron en diferentes puntos de los terrenos, separados por cinco metros, realizando un desplazamiento en zig-zag. De cada finca se tomaron diez muestras de suelo rizosférico, las cuales fueron homogeneizadas y divididas en tres submuestras compuestas que fueron almacenadas en refrigeración y transportadas al laboratorio para su análisis microbiológico. Para determinar las características fisicoquímicas de los suelos se tomó una muestra compuesta de cada terreno, y los resultados se pueden evidenciar en la tabla 1.

\subsection{Aislamiento de los hongos solubilizadores de fosfato}

Se realizó el aislamiento de hongos solubilizadores de fosfato por medio del método de diluciones seriadas y siembra en medio selectivo, utilizando el medio de cultivo NBRIP (Nacional Botanical Research Institute Phosphate growth medium) formulado por 
Tabla 1. Características fisicoquímicas de los suelos estudiados.

\begin{tabular}{llccccccccc}
\hline Finca & & \multicolumn{7}{c}{$\mathrm{P}(\mathrm{ppm})$} & \multicolumn{7}{c}{$\mathrm{meq} / 100 \mathrm{~g}$} \\
& Textura & $\mathrm{pH}$ & $\% \mathrm{MO}$ & $\mathrm{Bray} \mathrm{II}$ & $\mathrm{Al}$ & $\mathrm{Ca}$ & $\mathrm{Mg}$ & $\mathrm{K}$ & $\mathrm{Na}$ & $\mathrm{CIC}^{*}$ \\
\hline $\begin{array}{l}\text { El Recuerdo } \\
(1)\end{array}$ & $\begin{array}{l}\text { Franco } \\
\text { arcilloso } \\
\text { arenoso }\end{array}$ & 5.07 & 19.67 & 57.03 & 2.58 & 2.45 & 0.20 & 0.37 & 0.08 & 5.68 \\
$\begin{array}{l}\text { Villa Rosa } \\
(2)\end{array}$ & $\begin{array}{l}\text { Franco } \\
\text { arenoso }\end{array}$ & 4.72 & 13.45 & 37.76 & 2.22 & 2.09 & 0.37 & 0.22 & 0.13 & 5.04 \\
$\begin{array}{l}\text { Zarcillejos } \\
(3)\end{array}$ & Orgánico & 5.2 & 21.2 & 18.8 & 1.0 & 3.48 & 0.44 & 1.12 & 0.05 & 6.69 \\
$\begin{array}{l}\text { La Escuela } \\
(4)\end{array}$ & $\begin{array}{l}\text { Franco } \\
\text { arenoso }\end{array}$ & 5.2 & 15.7 & 60.5 & 1.0 & 2.04 & 0.16 & 0.11 & 0.07 & 3.38 \\
\hline
\end{tabular}

*CIC: capacidad de intercambio catiónico.

Nautiyal [9]. Este medio contiene (en g/L): Glucosa, 10; $\left(\mathrm{NH}_{4}\right)_{2} \mathrm{SO}_{4}, 0.1 ; \mathrm{KCl}, 0.2 ; \mathrm{MgSO}_{4} .7 \mathrm{H}_{2} \mathrm{O}, 0.25$; $\mathrm{MgCl}_{2} \cdot 6 \mathrm{H}_{2} \mathrm{O}, 5$; Agar, 14; $\mathrm{pH} 7$ enriquecido con fosfato tricálcico, $\mathrm{Ca}_{3} \mathrm{PO}_{4}$, al $0.5 \%$ como fuente de fosfato inorgánico e insoluble. Las cajas se incubaron a $28^{\circ} \mathrm{C}$ durante 14 días; al final de periodo de incubación se seleccionaron los hongos que mostraron actividad solubilizadora por la aparición de halos transparentes alrededor de las colonias [10]. Se realizó el proceso de purificación de los aislados y criopreservación a $-20^{\circ} \mathrm{C}$, usando glicerol al $50 \%$ como agente crioprotector [11].

\subsection{Evaluación cualitativa de la actividad solu- bilizadora de fosfato}

Las cepas de hongos solubilizadores criopreservadas fueron reactivadas, brindando las condiciones adecuadas de temperatura y tiempo de incubación ( $28^{\circ} \mathrm{C}$ durante 7 días) en medio de cultivo Agar papa dextrosa PDA (Oxoid®), con el objetivo de obtener buena cantidad de biomasa. Luego, se realizó un subcultivo por triplicado en medio de cultivo NBRIP enriquecido con fosfato tricálcico al $0.5 \%$, obteniendo así tres réplicas del halo de solubilización de cada aislado, para de esa forma determinar la eficiencia de solubilización de cada uno, según Seshadri et al. [12]. En este ensayo se utilizó como cepa control positivo a Aspergillus niger ATTC6295, ya que hace parte de un género que ha sido reportado como solubilizador de fosfato por la literatura $[4,10,14,15$, $16,17]$.

\subsection{Evaluación cuantitativa de la actividad solubilizadora de fosfato}

La evaluación desde el punto de vista cuantitativo se realizó por duplicado para cada hongo solubiliza- dor, utilizando el método del azul molibdeno, que es un procedimiento análogo al US Standart Methods 4500-P [13], adaptado a las condiciones del ensayo. Para esto se utilizó la prueba analítica Spectroquant $\AA$ Test fosfatos (Merck $\AA)$, por medio de la cual se puede determinar colorimétricamente la presencia de ortofosfatos en el medio de cultivo después del proceso de incubación. De cada aislado se tomaron tres cubos de micelio de $1 \mathrm{~cm}^{3}$ a partir de un cultivo de siete días de antigüedad, según metodología propuesta por Oliveira et al. [18] y Reddyet al. [17]; dichos cubos se inocularon en $50 \mathrm{~mL}$ de medio de cultivo NBRIP líquido y fueron incubados durante 14 días a $28^{\circ} \mathrm{C}$. Al final del proceso de incubación se centrifugaron los cultivos a $5000 \mathrm{rpm}$ durante cinco minutos, para poder medir el $\mathrm{pH}$ final del medio de cultivo según Charakborty et al. [10] y se tomaron alícuotas de $5 \mathrm{~mL}$ del sobrenadante, con el fin de realizar la prueba colorimétrica para la determinación de fosfatos solubles; se realizó la lectura en espectrofotómetro a $690 \mathrm{~nm}$ y se detectó la concentración de $\mathrm{mg} / \mathrm{L}$ de fosfato soluble, gracias a la curva de calibración realizada, usando como solución patrón $\mathrm{K}_{2} \mathrm{HPO}_{4}$ [19]. En el ensayo, la cepa control actuó como control positivo del proceso y el control negativo fue medio de cultivo sin inóculo.

\subsection{Identificación de aislados sobresalientes}

Los aislados fúngicos que presentaron mayor eficiencia de solubilización y mayor solubilización de fosfato en medio de cultivo líquido fueron identificados por medio de claves taxonómicas específicas hasta el nivel de género, según Gilman [20] y Watanabe [21]. 


\subsection{Análisis de resultados}

El análisis estadístico de los datos se realizó con ayuda de los programas MINITAB $\AA$ Release 14, Statistical Software y Statgraphics Plus versión 5.1. Debido a que los datos no cumplieron el supuesto de normalidad, se realizó la prueba no paramétrica Kruskal-Wallis por pares a un nivel de significancia del $P=0,05$ para verificar si existían diferencias estadísticamente significativas entre los aislamientos. Posteriormente se realizó la prueba LSD al 5\% de significancia para realizar las comparaciones múltiples entre los aislados en cuanto al diámetro de halo de solubilización, índice de eficiencia de solubilización, concentración de fosfato soluble en medio líquido y pH final del medio de cultivo. Se realizó una prueba de correlación de Pearson entre el pH del medio y la concentración de ortofosfatos presentes en el medio de cultivo al final del proceso de incubación para conocer la posible correlación entre estos parámetros.

\section{Resultados y discusión}

Se lograron aislar 43 hongos solubilizadores de fosfato a partir de las muestras de suelo rizosférico de las cuatro fincas cultivadas con papa (Solanum tuberosum); estas cepas fueron codificadas según la finca en la cual fueron encontradas, y evaluadas desde el punto de vista cualitativo y cuantitativo.

\subsection{Evaluación cualitativa de la actividad solubilizadora de fosfato}

En esta investigación se observaron halos de solubilización que oscilaron entre $0.7 \mathrm{~mm}$, para el aislamiento 1-HSF-05, y $12 \mathrm{~mm}$, para el aislado 4-HSF07, que es mucho mayor al mostrado por el control positivo cepa A. niger ATTC6295, cuyo halo de solubilización fue de $4.3 \mathrm{~mm}$ (tabla 2). Pérez et al. [23] indican que la variación de la proporción entre el tamaño de halo y el tamaño de la colonia sugiere la existencia de diferentes grados de eficiencia de solubilización de fosfatos. Los valores reportados por los aislamientos evaluados son superiores a los reportados por Moratto et al. [24], quien evaluó la capacidad solubilizadora de hongos filamentosos aislados del páramo de Guerrero, en Cundinamarca, y encontró halos de solubilización entre 1 y $3.25 \mathrm{~mm}$.
En este estudio se evidenció la pérdida de la actividad solubilizadora en términos de halo de solubilización por parte de algunos aislados después del proceso de reactivación, como es el caso de los aislamientos con código 1-HSF-08, 1-HSF-10, 1-HSF-16, 3-HSF-05 y 3-HSF-10; en algunos estudios ha sido reportada la inestabilidad del carácter solubilizador en algunas cepas después de varios ciclos de inoculación $[22,15,17]$.

El parámetro de la eficiencia de solubilización propuesto por Seshadri et al. [12] es importante, ya que indica cuáles aislamientos muestran las mejores características solubilizadoras desde el punto de vista funcional, ya que un aislado que muestre alta eficiencia usa para sustentar su crecimiento menos fosfato del que solubiliza, dejando disponible para la planta la mayor parte de este fosfato. En cuanto a este parámetro para los aislados en estudio, se evidenciaron valores entre $3.3 \%$, para el hongo 1-HSF-05, y $200 \%$, para el aislado con código 4-HSF-10; el control positivo en este caso mostró una eficiencia del $28.9 \%$. Estos son valores superiores a los reportados por Moratto et al. [24], quienes reportaron eficiencias de solubilización, para hongos solubilizadores, entre 2.94 y $17.02 \%$.

\subsection{Evaluación cuantitativa de la actividad solubilizadora de fosfato}

Como se indicó en la evaluación cualitativa de la actividad solubilizadora de fosfatos, algunos aislados fúngicos no exhibieron halos de solubilización después de ser reactivados; es conocido que la confiabilidad de la técnica basada en el criterio del halo de solubilización es cuestionable, ya que algunos aislados que no producen halo en placas de agar pueden solubilizar varios tipos de fosfatos insolubles en medio líquido [25]. Nautiyal [9] comprobó que el método cuantitativo es más sensible, y por eso es recomendable a la hora de evaluar cualquier microorganismo con potencial solubilizador. Por esta razón, se decidió evaluar cuantitativamente a todos los aislamientos criopreservados, para verificar si realmente perdieron su actividad o solo la muestran en medios de cultivo líquidos.

En este análisis se pudo evidenciar que prácticamente todos los aislados criopreservados exhibieron actividad solubilizadora en medio de cultivo líquido, a pesar de que algunos no habían mostrado halo de 
Tabla 2. Tamaño de halo y eficiencia de solubilización de los hongos solubilizadores de fosfato obtenidos en las cuatro fincas cultivadas con papa (Solanum Tuberosum), localizadas en el páramo de Rabanal.

\begin{tabular}{|c|c|c|c|c|c|c|c|}
\hline Aislamiento & $\begin{array}{l}\text { Diámetro } \\
\text { de la } \\
\text { colonia } \\
(\mathrm{mm})\end{array}$ & $\begin{array}{l}\text { Diámetro } \\
\text { del halo } \\
\text { de solubi- } \\
\text { lización } \\
(\mathrm{mm})\end{array}$ & $\begin{array}{l}\text { Índice de efi- } \\
\text { ciencia }(\%)\end{array}$ & Aislamiento & $\begin{array}{l}\text { Diámetro } \\
\text { de la } \\
\text { colonia } \\
(\mathrm{mm})\end{array}$ & $\begin{array}{l}\text { Diámetro del } \\
\text { halo de so- } \\
\text { lubilización } \\
(\mathrm{mm})\end{array}$ & $\begin{array}{l}\text { Índice de efi- } \\
\text { ciencia }(\%)\end{array}$ \\
\hline 1-HSF-01 & 25.0 & $6.7_{g h i}$ & $26.1_{e f g h i}$ & 3-HSF-02 & 11.0 & $5.0_{e f g}$ & $51.3_{k l}$ \\
\hline 1-HSF-02 & 24.3 & $6.0_{e f g h}$ & $24.8_{\text {efghi }}$ & 3-HSF-03 & 30.3 & $6.0_{e f g h}$ & $19.9_{\text {cdefg }}$ \\
\hline 1-HSF-03 & 21.7 & $5.0_{e f g}$ & 23.3 defghi & 3-HSF-04 & 31.3 & $6.0_{e f g h}$ & $19.4_{c d e f g}$ \\
\hline 1-HSF-04 & 17.3 & $6.3_{f g h}$ & $38.1_{i j k}$ & 3-HSF-05 & 58.7 & $0.0_{a}$ & $0.0_{a}$ \\
\hline 1-HSF-05 & 16.7 & $0.7_{a b}$ & $3.3_{a b}$ & 3-HSF-06 & 29.3 & $6.7_{g h i}$ & 22.7 defghi \\
\hline 1-HSF-06 & 20.3 & $4.7_{\text {defg }}$ & $22.6_{d e f g h}$ & 3-HSF-07 & 34.7 & $6.0_{e f g h}$ & $17.5_{b c d e f g}$ \\
\hline 1-HSF-07 & 23.0 & $5.0_{e f g}$ & $21.8_{\text {cdefgh }}$ & 3-HSF-08 & 27.3 & $6.7_{g h i}$ & $24.2_{\text {efghi }}$ \\
\hline 1-HSF-08 & 29.3 & $0.0_{a}$ & $0.0_{a}$ & 3-HSF-09 & 38.7 & $6.7_{g h i}$ & 17.2 bcdefg \\
\hline 1-HSF-09 & 25.3 & $5.0_{e f g}$ & $20.2_{\text {cdefgh }}$ & 3-HSF-10 & 34.7 & $0.0_{a}$ & $0.0_{a}$ \\
\hline 1-HSF-10 & 28.3 & $0.0_{a}$ & $0.0_{a}$ & 3-HSF-11 & 36.7 & $8.0_{h i}$ & $21.7_{\text {cdefgh }}$ \\
\hline 1-HSF-11 & 14.3 & $5.0_{e f g}$ & $35.4_{h i j}$ & 4-HSF-01 & 40.0 & $5.3_{e f g}$ & $16.3_{b c d e f g}$ \\
\hline 1-HSF-12 & 22.3 & $4.0_{c d e}$ & $18.4_{b c d e f g}$ & 4-HSF-02 & 50.0 & $6.0_{\text {efgh }}$ & $12.0_{a b c d e}$ \\
\hline 1-HSF-13 & 10.0 & $4.3_{d e f}$ & $43.3_{j k l}$ & 4-HSF-03 & 13.3 & $1.7_{a b}$ & $15.0_{a b c d e f}$ \\
\hline 1-HSF-14 & 15.0 & $2.0_{a b c}$ & 14.4abcdef & 4-HSF-04 & 17.3 & $2.7_{b c d}$ & 15.4abcdef \\
\hline 1-HSF-15 & 23.3 & $5.0_{e f g}$ & $21.7_{\text {cdefgh }}$ & 4-HSF-05 & 34.7 & $6.7_{g h i}$ & $19.7_{\text {cdefg }}$ \\
\hline 1-HSF-16 & 20.0 & $0.0_{a}$ & $0.0_{a}$ & 4-HSF-06 & 50.0 & $4.0_{c d e}$ & $8.0_{a b c d}$ \\
\hline 1-HSF-17 & 17.3 & $2.0_{a b c}$ & $11.7_{a b c d e}$ & 4-HSF-07 & 75.7 & $12.0_{j}$ & $15.8_{a b c d e}$ \\
\hline 2-HSF-01 & 20.0 & $4.7_{\text {defg }}$ & $23.3_{\text {defghi }}$ & 4-HSF-08 & 60.0 & $4.0_{c d e}$ & $6.7_{a b c}$ \\
\hline 2-HSF-02 & 15.7 & $8.7_{i}$ & $55.7_{l}$ & 4-HSF-09 & 30.7 & $4.0_{c d e}$ & 18.2 $b c d e f g$ \\
\hline 2-HSF-03 & 20.0 & $4.0_{c d e}$ & $20.0_{c d e f g h}$ & 4-HSF-10 & 4.0 & $8.0_{h i}$ & $200.0_{m}$ \\
\hline 2-HSF-04 & 11.7 & $1.7_{a b}$ & 14.4 abcdef & 4-HSF-11 & 23.3 & $6.7_{g h i}$ & 31.3 ${ }_{g h i j}$ \\
\hline 3-HSF-01 & 30.3 & $6.0_{e f g h}$ & $20.5_{\text {cdefgh }}$ & Control + & 15.0 & $4.3_{\text {def }}$ & $28.9_{f g h i j}$ \\
\hline
\end{tabular}

HSF: Hongo solubilizador de fosfato tricálcico al 0,5\%. Diámetro de colonia, tamaño de halo y eficiencia de solubilización es promedio de tres réplicas. Control +: A.niger ATTC6295. Letras iguales no difieren significativamente, según la prueba de LDS al $5 \%$.

solubilización en medio sólido después de la reactivación. Pero contradictoriamente, el hongo 3-HSF-03, que inicialmente había mostrado halo de solubilización en medio de cultivo sólido, no solubilizó fosfato en medio líquido; esto pudo deberse a que la actividad solubilizadora puede perderse tras sucesivos subcultivos; además, en estudios previos se ha reportado que en algunos casos se presentan resultados contradictorios entre la detección del halo en medio sólido y la solubilización en medio de cultivo líquido $[26,27,28]$.

Las actividades solubilizadoras de los hongos en estudio oscilaron entre $30.3 \mathrm{mgPO}_{4} / \mathrm{L}$, para el aislamiento 3-HSF-04, y $1370.6 \mathrm{mgPO}_{4} / \mathrm{L}$, para el aislado 2-HSF-02, cuya concentración es superior a la reportada para el control positivo del experimento, que fue de $1073.3 \mathrm{mgPO}_{4} / \mathrm{L}$ (tabla 3). Los valores tan altos registrados por los aislamientos de este estudio podrían explicarse por el tiempo de incubación de estos ensayos, que fue de 14 días, mayor al reportado por otros estudios que evaluaron la cantidad de fosfato solubilizado por microorganismos en dos o diez días [9, 29].

Las concentraciones de ortofosfatos liberados tras el proceso de incubación de estos aislamientos son muy variables; esto podría explicarse por sus diferentes capacidades solubilizadoras, que se relacionan con la variación en las tasas de difusión de ácidos orgánicos secretados por estos microorganismos y que pueden acidificar el medio de cultivo [9] o por la complejidad estructural y el tamaño de la partícula de fosfato [19].

Los resultados obtenidos en esta investigación son superiores a los reportados por Beltrán [30], quien evaluó la capacidad solubilizadora de hongos aislados de suelos arroceros en el departamento del Meta (Colombia), encontrando actividades de hasta 332.6 $\mathrm{mgPO}_{4} / \mathrm{L}$ en el mismo periodo de incubación. Sin embargo, Chakraborty et al. [10], evaluando la capacidad solubilizadora de hongos aislados de campos agrícolas, bosques y cuencas de ríos en el norte de 
Bengala (India), encontraron concentraciones de fósforo soluble después de diez días de incubación de hasta $856 \mathrm{mg} / \mathrm{L}$, valores comparables con los reportados por algunos aislamientos en este estudio.

En el ensayo de solubilización en medio líquido, el medio de cultivo tenía un $\mathrm{pH}$ inicial de 7.0, y al final del proceso de incubación se pudo evidenciar que la totalidad de los aislamientos acidificaron el medio de cultivo; estos resultados indicarían una relación entre la acidez del medio y la liberación de fosfatos solubles, resultados ya encontrados por otros investigadores [24, 4, 19, 30, 29]. Sin embargo, el análisis de correlación indicó que no existe una relación lineal estadísticamente significativa entre el pH final del medio de cultivo y la concentración de ortofosfatos en el medio después del proceso de incubación (r: -0,180 y p: 0,094).

Así mismo, Narsian et al. [16] y Reddy et al. [17] indicaron que no se puede establecer una relación significativa entre la cantidad de fosfato solubilizado y la caída del $\mathrm{pH}$ del medio, ya que se conoce que un $\mathrm{pH}$ alto en el medio de cultivo puede favorecer la actividad de la glucosa oxidasa [4], que es la principal enzima implicada en la solubilización de fosfatos. De igual forma, Oliviera et al. [18] observaron una baja correlación entre la reducción del pH y el aumento en el fósforo soluble en el medio de cultivo después de diez días de incubación, indicando que la disminución en la concentración de fósforo insoluble puede ser influenciada por otros factores.

Si bien es reconocido que el principal mecanismo de solubilización es la producción de ácidos orgánicos, y que los hongos filamentosos son productores de dichos metabolitos [31, 17], se han propuesto otros posibles mecanismos de solubilización, pues en muchas ocasiones no existe una correlación lineal entre el $\mathrm{pH}$ y la cantidad de fosfato solubilizado en los medios de cultivo, como es el caso de este estudio $[32,16,17]$. Algunos investigadores han propuesto como alternativa de solubilización que algunos microorganismos pueden producir sustancias quelantes o liberar al medio extracelular ácidos de tipo inorgánico, como el sulfúrico, el nítrico y el carbónico [27].

\subsection{Identificación de los aislamientos sobresalientes}

Los mejores aislamientos se seleccionaron según dos criterios: el primero es la mayor eficiencia de solubilización de acuerdo con la relación entre el tamaño de halo y el tamaño de la colonia, y el segundo es la mayor cantidad de fosfato solubilizado en medio líquido. El mejor hongo solubilizador, según la eficiencia de solubilización, fue el aislamiento con código 4-HSF-10, que corresponde al género Scopuraliopsis sp.; este hongo es un deuteromiceto que es saprófito común de suelos y ha sido aislado de variedad de sustratos; por lo tanto, es un organismo cosmopolita [33]. Este género ha sido reportado anteriormente como solubilizador de fosfato en suelos del trapecio amazónico [34], y también se encontró como hongo solubilizador de la rizósfera de melón (Cucumis melo L. cv. gold mine) en suelos brasileros [35]; adicionalmente, es reconocido como hongo endofítico, es decir, que puede desarrollar relaciones mutualistas planta-microorganismo, actuando como antagonista contra plagas y enfermedades [36].

Finalmente, el mejor hongo en términos de capacidad de solubilización de fosfato en medio de cultivo líquido corresponde al aislamiento con código 2HSF-02; se trata de un hongo del género Penicillium sp., cuyas poblaciones están comúnmente asociadas a la rizósfera de papa [37], pero también son ampliamente reconocidas por solubilizar fosfatos minerales en varios tipos de suelo [10, 15, 17, 18, 24, 34, 38, 39, $40,41,42]$. Un inóculo comercial a base del hongo Penicillium bilaii está disponible en Norte América, con buenos resultados a la hora de incrementar la absorción de P por la planta [43], y en la actualidad, en Colombia se produce un biofertilizante líquido a base del hongo Penicillium janthinellum, como producto de la investigación que realiza el Instituto de Biotecnología de la Universidad Nacional de Colombia, sede Bogotá, con buenos resultados, dado que incrementa el rendimiento de un 5 a un $38 \%$ en cultivos de arroz [44].

\section{Conclusiones}

El presente estudio permitió evidenciar la presencia de hongos solubilizadores de fosfato en los suelos de páramo estudiados. Kucey [22], encontró una correlación entre el número de hongos solubilizadores de fosfato y los niveles de fósforo total en los suelos, 
Tabla 3. Cuantificación de fosfato solubilizado en medio líquido por parte de los aislados fúngicos obtenidos en las cuatro fincas cultivadas con papa (Solanum Tuberosum) localizadas en el páramo de Rabanal.

\begin{tabular}{|c|c|c|c|c|c|}
\hline AISLADO & $\mathrm{pH}$ & {$\left[\mathrm{PO}_{4}\right]$ en $\mathrm{mg} / \mathrm{L}$} & AISLADO & $\mathrm{pH}$ & {$\left[\mathrm{PO}_{4}\right]$ en $\mathrm{mg} / \mathrm{L}$} \\
\hline 1-HSF-01 & $4.0_{b}$ & $887.4_{\text {ghijk }}$ & 3-HSF-02 & $4.0_{b}$ & $276.1_{a b c d e}$ \\
\hline 1-HSF-02 & $4.5_{a b}$ & $135.3_{a b c}$ & 3-HSF-03 & $4.5_{a b}$ & $0.0_{a}$ \\
\hline 1-HSF-03 & $4.0_{b}$ & $28.4_{a b c d e}$ & 3-HSF-04 & $5.0_{b}$ & $30.3_{a b}$ \\
\hline 1-HSF-04 & $4.5_{a b}$ & $136.4_{a b c}$ & 3-HSF-05 & $4.5_{a b}$ & $157.4_{a b c d}$ \\
\hline 1-HSF-05 & $4.0_{b}$ & $372.7_{a b c d e f}$ & 3-HSF-06 & $5.0_{b}$ & $191.0_{a b c d e}$ \\
\hline 1-HSF-06 & $3.0_{a}$ & 613.3. cdefghi & 3-HSF-07 & $5.0_{b}$ & 218.3 abcde \\
\hline 1-HSF-07 & $4.5_{a b}$ & 401.1 $1_{a b c d e f g}$ & 3-HSF-08 & $5.0_{b}$ & 793.9 fghij \\
\hline 1-HSF-08 & $4.5_{a b}$ & $244.6_{a b c d e}$ & 3-HSF-09 & $5.0_{b}$ & $390.6_{a b c d e f}$ \\
\hline 1-HSF-09 & $5.0_{b}$ & 259.2 abcde & 3-HSF-10 & $5.0_{b}$ & $330.7_{a b c d e f}$ \\
\hline 1-HSF-10 & $4.0_{b}$ & $216.2_{a b c d e}$ & 3-HSF-11 & $4.5_{a b}$ & $131.1_{a b c}$ \\
\hline 1-HSF-11 & $4.0_{b}$ & 343.3 abcdef & 4-HSF-01 & $4.0_{b}$ & $649.0_{\text {defghij }}$ \\
\hline 1-HSF-12 & $3.0_{a}$ & $578.6_{c d e f g h}$ & 4-HSF-02 & $5.0_{b}$ & $487.2_{b c d e f g h}$ \\
\hline 1-HSF-13 & $4.0_{b}$ & $675.2_{\text {efghij }}$ & 4-HSF-03 & $5.0_{b}$ & $66.0_{a b}$ \\
\hline 1-HSF-14 & $3.0_{a}$ & $158.4_{a b c d}$ & 4-HSF-04 & $5.0_{b}$ & $166.9_{a b c d}$ \\
\hline 1-HSF-15 & $3.0_{a}$ & 901.1 ${ }_{h i j k}$ & 4-HSF-05 & $4.0_{b}$ & $500.9_{b c d e f g h}$ \\
\hline 1-HSF-16 & $4.5_{a b}$ & $464.1_{a b c d e f g h}$ & 4-HSF-06 & $4.5_{a b}$ & $570.2_{\text {cdefgh }}$ \\
\hline 1-HSF-17 & $4.5_{a b}$ & $201.5_{a b c d e}$ & 4-HSF-07 & $5.0_{b}$ & $499.8_{b c d e f g h}$ \\
\hline 2-HSF-01 & $5.0_{b}$ & $445.2_{a b c d e f g h}$ & 4-HSF-08 & $5.0_{b}$ & $253.0_{a b c d e}$ \\
\hline 2-HSF-02 & $4.5_{a b}$ & $1370.6_{k}$ & 4-HSF-09 & $5.0_{b}$ & 293.9 abcde \\
\hline 2-HSF-03 & $5.0_{b}$ & $1125.9_{j k}$ & 4-HSF-10 & $5.0_{b}$ & $133.2_{a b c}$ \\
\hline 2-HSF-04 & $4.5_{a b}$ & $1122.7_{j k}$ & 4-HSF-11 & $4.5_{a b}$ & 210.9 abcde \\
\hline 3-HSF-01 & $5.0_{b}$ & $69.2_{a b}$ & Control + & $5.0_{b}$ & $1073.3_{i j k}$ \\
\hline
\end{tabular}

infiriendo que los hongos solubilizadores podrían favorecer el incremento y la productividad de las plantas asociadas. Se lograron aislar y caracterizar 43 hongos solubilizadores de fosfato a partir de la rizósfera de plantas de papa (Solanum tuberosum) y se seleccionaron dos cepas promisorias para la fabricación de biofertilizantes, desde el punto de vista de la solubilización de fosfato, pertenecientes a los géneros Scopuraliopsis sp. y Penicillium sp., ya reconocidos por solubilizar fosfato en diferentes tipos de ecosistemas $[10,15,17,18,24,34,38,39,40$, $41,42]$. Finalmente, a pesar de que los hongos tienen una capacidad inferior de colonizar las raíces de plantas, en comparación con las bacterias, ellos son más acidotolerantes; por esta razón tienen un mejor potencial para ser usados como agentes que convierten fósforo inorgánico e insoluble a formas solubles utilizables por las plantas en suelos ácidos [4], como los suelos en estudio. Es necesario realizar evaluaciones a nivel de invernadero y de campo con los dos aislamientos promisorios, para conocer cuál de los dos muestra una actividad solubilizadora constante y tiene efectos de promoción de crecimiento vegetal en plantas de papa (Solanum tuberosum); esos resultados indicarían cuál hongo podría convertirse en una futura cepa comercial para la fabricación de biofertilizantes.

\section{Agradecimientos}

Al laboratorio de Microbiología de la Universidad de Boyacá; a la bacterióloga, M. Sc. en Microbiología, Jenny Carolina Hernández Barrera, por su colaboración con la identificación de los aislamientos promisorios. Finalmente, al Grupo de Investigación Gestión Ambiental, de la Facultad de Ciencias e Ingeniería de la Uniboyacá.

\section{Referencias}

[1] Ministerio de Agricultura y Desarrollo Rural, observatorio Agrocadenas Colombia, Documento de trabajo no. 54, La cadena de la papa en Colombia, una mirada global de su estructura y dinámica 1991-2005, 2005. Disponible en: http://www.agronet.gov.co/www/docs_agronet/ 2005112163731_caracterizacion_papa.pdf

[2] M. Beare, M. Reddy, G. Tian, and S. Srivastava, "Agricultural intensification, soil biodiversity 
and agroecosistem function in the tropics: the role of descomposer biota", App. Soil. Ecol., vol. 6, pp. 87-108, 1997.

[3] Corporación Autónoma de Boyacá (Corpoboyacá), Corporación Autónoma de la Represa de Chivor (Corpochivor), Plan de acción Páramo Rabanal 2005-2010, Documento Técnico, 2005. Disponible en: http://www.condesan.org/ppa/sites/default/files/ recursos/archivos/Documento \%20soporte \%20 declaratoria \%20Rabanal.pdf

[4] C. Chuang, Y. Kno, Ch. Chao, and W. Chao, "Solubilization of inorganic phosphates and plant growth promotion by Aspergillus niger", Biol. Fertil. Soils., vol. 43, pp. 575-584, 2007.

[5] H. Lambers, M. Shane, M. Cramer, S. Pearse, and E. Veneklaas, "Root structure and functioning for efficient acquisition of phosphorus: matching morphological and physiological traits", Annals. Bot., vol. 98, pp. 693-713, 2006.

[6] Ç. Kucuk, M. Kivang, E. Kinaci, and G. Kinaci, "Determination of the growth and solubilization capabilities of Thichoderma harzianum T1", Biologia, vol. 63, no. 2, pp. 167-170, 2008.

[7] L. Castilla, "La biofertilización en el manejo integrado de nutrimentos para la nutrición vegetal", en: Biofertilización: una alternativa viable para la nutrición vegetal. Sociedad Colombiana de la Ciencia del Suelo. Capítulo Tolima, pp. 7-16, 2006.

[8] H. Fankem, D. Nwaga, A. Deubel, W. Dieng, and W. Merbach, "Occurrence and functioning of phosphate solubilizing microorganisms from oil palm tree (Elaeis guineensis) rhizosphere in Cameroon", African Journal of Biotechnology, vol. 5, no. 24, pp. 2450-2460, 2006.

[9] C. Nautiyal, "An efficient microbiological growth medium for screening phosphatesolubilizing microorganisms", FEMS Microbiology Letters, vol. 170, pp. 265-270, 1999.

[10] B. Chakraborty, U. Chakraborty, A. Sha, K. Sunar, and P. Dey, "Evaluation of phosphate solubilizers from soils of North bengal and their diversity analysis", World Journal of Agricultural Sciences, vol. 6, no. 2, pp. 195-200, 2010.
[11] Y. Gato, "Métodos de conservación y formulación de Trichoderma harzianum rifai", Fitosanidad, vol. 14, no. 3, pp. 189-195, 2010.

[12] S. Seshadri, R. Muthukumarasamy, C. Lakshminarasimhan, and S. Ignacimuthu, "Solubilization of inorganic phosphates by Azospirillum halopraeferans", Current Science, vol. 79, no. 5, pp. 565-567, 2000.

[13] APHA, Standard Methods for the Examination of Water and Wastewater, 22th ed. Washington: American Public Health Association, DC, 2012.

[14] N. Vassilev, M. Vassileva, and M. Spassova, "Production of gluconic acid by Aspegillus niger inmovilized in polyurethane foam", Appl. Microbial. Biotechnol., vol. 39, pp. 285-288, 1993.

[15] P. Illmer, and F. Schinner, "Solubilization of inorganic calcium phosphates-solubilization mechanisms", Soil. Biol. Biochem, vol. 27, no. 3, pp. 257-263, 1995.

[16] V. Narsian, and H. Patel, "Aspergillus aculeatus as a rock phosphate solubilizers", Soil Biol. Biochem., vol. 32, pp. 559-565, 2000.

[17] M. Reddy, S. Kumar, K. Babita, and M. Reddy, "Biosolubilization of poorly soluble rock phosphates by Aspergillus tubingensis and Aspergillus niger", Bioresource Technology, vol. 84, pp. 187-189, 2002.

[18] C. Oliviera, V. Alves, I. Marriel, E. Gómez, M. Scotti, M. Carneiro, M. Guimaraes, R. Schaffert, and N. Sa, "Phosphate solubilizing microorganisms isolated from rhizosphere of maize cultivated in an oxisol of the Brazilian Cerrado Biome", Soil. Biol. Biochem., vol. 41, no. 9, pp. 1782-1787, 2009.

[19] P. Naik, G. Raman, K. Narayanan, and N. Natarajan, "Assesment of genetic and functional diversity of phosphate solubilizing fluorescent pseudomonads isolated from rhizospheric soil", BCM Microbiology, vol. 8, no. 230, pp. 1-14, 2008.

[20] J. Gilman, A Manual of soil fungi, 2. ed. Iowa State University Press: Edit. Ames, 1966.

[21] T. Watanabe, Pictorial atlas of soil and seed fungi. Morphologies of cultured fungi and key to species. Second edition. CRC press. Boca 
Ratón, London, New York, Washington D.C., 2002.

[22] R. Kucey, "Phosphate-solubilizing bacteria and fungi in various cultivated and virgin Alberta soils", Canadian Journal Soil Science, vol. 63, pp. 671-678, 1983.

[23] E. Pérez, M. Sulbaran, M. Ball, and L. Yarzabal, "Isolation and characterization of mineral phosphate-solubilizing bacteria naturally colonizing a limonitic crust in the south-eastern Venezuelan region", Soil. Biol. Biochem., vol. 39, pp. 2905-2914, 2007.

[24] C. Moratto, L. Martínez, H. Valencia, and J. Sánchez, "Efecto del uso del suelo sobre hongos solubilizadores de fosfato y bacterias diazotróficas en el páramo de Guerrero (Cundinamarca)", Agronomía Colombiana, vol. 23, no. 2, pp. 299-309, 2005.

[25] R. Gupta, R. Singal, A. Shankar, R. Kuhad, and R. Saxena, "A modified plate assay for screening phosphate solubilizing microorganisms", J. Gen. Appl. Microbiol, vol. 40, pp. 255-260, 1994.

[26] K. Ostwal, V. Bhide, "Solubilization of tricalcicum phosphate by soil Pseudomonas", Indian. J. Exp. Biol, vol. 10, pp. 153-164, 1972.

[27] H. Rodríguez, and R. Fraga, "Phosphate solubilizing bacteria and their role in plant growth promotion", Biotechnology Advances, vol. 17, pp. 319-339, 1999.

[28] M. Whitelaw, T. Harden, and K. Helyar, "Phosphate solubilization in solution culture by the soil fungus Penicillium radicum", Soil. Biol. Biochem., vol. 31, pp. 655-665, 1999.

[29] S. Banerjee, R. Palit, C. Sengupta, and D. Standing, "Stress induced phosphate solubilization by Arthrobacter sp. and Bacillus sp. isolated from tomato rhizosphere", Australian Journal of crop science AJCS, vol. 4, no. 6, pp. 378-383, 2010.

[30] M. Beltrán, "Evaluación del efecto del sistema de producción del cultivo del arroz (secano e inundado) sobre la población microbiana y la actividad enzimática asociada al metabolismo edáfico del fósforo", Tesis de Maestría, Facultad de Ciencias, Universidad Nacional de Colombia, Sede Bogotá, 2009.
[31] M. Matty, "The production of organic acids", Rev. Biotechnol., vol. 12, pp. 87-132, 1992.

[32] N. Ahmad, and K. Jha, "Solubilization of rock phosphate by microorganism isolated from Bihar soil", J. Can. Appl. Microbiol., vol. 14, pp. 89-95, 1968.

[33] M. Estrella, A. Gómez, E. Mellado, M. Buitrago, A. Monzóna, and J. Rodríguez, "Scopulariopsis brevicaulis, a fungal pathogen resistant to broad-spectrum antifungal agents", Antimicrobial Agents and Chemotherapy, vol. 47, no. 7, pp. 2339-2341, 2003.

[34] Y. Useche, H. Valencia, and H. Pérez, "Caracterización de bacterias y hongos solubilizadores de fosfato bajo tres usos de suelo en el sur del trapecio amazónico", Acta Biológica Colombiana, vol. 9, no. 2, pp. 42-45, 2004.

[35] F. Coutinho, M. de Queiroz, and A. Mayumi, "Phosphate-solubilizing fungi isolated from a semiarid área cultivated with melon (Cucumis melo L. cv. gold mine)", Acta Botanica Brasilica, vol. 25, no. 4, pp. 929-931, 2011.

[36] M. Barrios, "Estudio de hongos endofíticos como inductores de resistencia para el control de Sigatoka negra (Mycosphaerella fijiensis morelet) en plátano". Tesis para optar el título de Magister Scientiae en Agricultura Ecológica, CATIE (Centro Agronómico Tropical de Investigación y Enseñanza), 2006.

[37] R. Larkin, "Characterizatión of soil microbial communities under different potato cropping systems by microbial population dynamics, substrate utilization, and fatty acid profiles", Soil. Bio. Biochem., vol. 35, pp. 1451-1466, 2003.

[38] A. Goldstein, "Bacterial solubilization of mineral phosphates: historical perspectives and future prospects", Am. J. Altern. Agric., vol. 1, pp. 51-57, 1986.

[39] P. Illmer, A. Barbato, and F. Schinner, "Solubilization of hardly soluble ALPO4 with psolubilizing microorganisms", Soil. Biol. Biochem., vol. 27, no. 3, pp. 265-270, 1994.

[40] P. Asea, R. Kucey, and J. Stewart, "Inorganic phosphate solubilization by two Penicillium species in solution culture and in soil", Soil Biol. Biochem., vol. 20, pp. 459-464, 1988. 
[41] R. Kucey, "Effect of Penicillium billai on the solubility and uptake of $\mathrm{P}$ and micronutrients from soil by wheat", Can. J. Soil Sci., vol. 68, pp. 261-270, 1988.

[42] P. Gyaneshwar, G. Naresh, L. Kumar, J. Parekh, and P. Poole, "Role of soil microorganisms in improving P nutrition of plants", Plant and Soil, vol. 245, pp. 83-93, 2002.

[43] N. Osorio, "Microorganismos del suelo y su efecto sobre la disponibilidad de nutrientes en suelos ácidos del trópico”, Suelos Ecuatoriales, vol. 41, no. 1, pp. 74-91, 2010.

[44] N. Moreno, D. Uribe, "Biofertilizantes para la agricultura en Colombia". En: M. Izaguirre, C. Labandera, J. Sanjuan (Eds.): Biofertilizantes en Iberoamérica: Visión técnica, científica y empresarial. Vol. 1. Montevideo: Denad Internacional, pp. 38-45. 